\title{
Gestação e cuidado em saúde: Narrativas de adolescentes gestantes na comunidade
}

\section{da Rocinha-RJ}

\author{
Management and health care: Narratives of pregnant adolescents in the community of Rocinha-RJ \\ Embarazo y atención médica: Narrativas de adolescentes embarazadas en la comunidad de
}

Rocinha-RJ

Recebido: 05/07/2021 | Revisado: 10/07/2021 | Aceito: 11/07/2021 | Publicado: 21/07/2021

\author{
Walquiria Baihense de Araújo Couto \\ ORCID: https://orcid.org/0000-0002-9643-6356 \\ Universidade Federal do Estado do Rio de Janeiro, Brasil \\ E-mail walquiria.couto@gmail.com \\ Simone Mendes Carvalho \\ ORCID: https://orcid.org/0000-0001-8821-9725 \\ Universidade Federal do Estado do Rio de Janeiro, Brasil \\ E-mail: simone.carvalho@unirio.br \\ Ana Claudia Barros Pinnas \\ ORCID: https://orcid.org/0000-0003-4964-6681 \\ Universidade Federal do Estado do Rio de Janeiro, Brasil \\ E-mail: anapinnas@gmail.com
}

\begin{abstract}
Resumo
Este estudo tem como objetivo identificar o significado da gestação e do cuidado em saúde sob a ótica de adolescentes gestantes, durante o pré-natal, no contexto geográfico, cultural e social do bairro da Rocinha/RJ. A metodologia utilizada foi de pesquisa qualitativa, exploratória, fundamentada nos delineamentos da pesquisa social. Foram entrevistadas 20 adolescentes gestantes, na faixa de 10 a 19 anos. A coleta dos dados foi por meio de um roteiro de entrevista semiestruturado e para a análise, utilizou-se a técnica de conteúdo temático categorial proposta por Bardin e Oliveira. Acerca dos resultados, surgiram categorias identificadas pelo método de análise de conteúdo, e uma destas categorias se refere a visão sobre gravidez e cuidado das adolescentes gestantes. Levando-se em consideração que a visão de cuidado é sempre subjetiva e única, estas jovens demonstraram que a forma como são acolhidas na unidade de saúde onde realizam o pré-natal é o diferencial para iniciar seu pré-natal e dar continuidade ao seu acompanhamento. Inseridas em um contexto de vulnerabilidades, muitas destas adolescentes esperam, por meio da gestação, uma forma de reconhecimento e inserção na sociedade, de valorização e pertencimento e exprimem das formas mais variadas, a preocupação e zelo consigo mesmas e com o outro (no caso o filho), sendo promotoras do seu processo de cuidado juntamente com os profissionais de saúde que as assistem.
\end{abstract}

Palavras-chave: Adolescentes gestantes; Cuidado em saúde; Atenção básica; Vulnerabilidades; Acolhimento.

\begin{abstract}
This study aims to identify the meaning of pregnancy and health care from the perspective of pregnant adolescents, during prenatal care, in the geographic, cultural and social context of the Rocinha/RJ neighborhood. The methodology used was qualitative, exploratory research, based on the outlines of social research. Twenty pregnant teenagers aged between 10 and 19 years were interviewed. Data collection was through a semi-structured interview script and for the analysis, the categorical thematic content technique proposed by Bardin and Oliveira was used. Regarding the results, categories identified by the method of content analysis emerged, and one of these categories refers to the view on pregnancy and care of pregnant adolescents. Taking into account that the vision of care is always subjective and unique, these young women demonstrated that the way they are welcomed at the health unit where they perform prenatal care is the differential for starting their prenatal care and continuing their follow-up. Inserted in a context of vulnerabilities, many of these teenagers expect, through pregnancy, a form of recognition and insertion in society, of valuing and belonging, and they express, in the most varied ways, the concern and zeal for themselves and for the other (in this case the child), being promoters of their care process together with the health professionals who assist them.
\end{abstract}

Keywords: Pregnant Adolescents; Health care; Basic attention; Vulnerabilities; Host.

\section{Resumen}

Este estudio tiene como objetivo identificar el significado del embarazo y la atención de la salud desde la perspectiva de las adolescentes embarazadas, durante la atención prenatal, en el contexto geográfico, cultural y social del barrio Rocinha/RJ. La metodología utilizada fue la investigación cualitativa, exploratoria, basada en los lineamientos de la 
investigación social. Se entrevistó a 20 adolescentes embarazadas de entre 10 y 19 años. La recolección de datos se realizó a través de un guión de entrevista semiestructurado y para el análisis se utilizó la técnica de contenido temático categórico propuesta por Bardin y Oliveira. En cuanto a los resultados, surgieron categorías identificadas por el método de análisis de contenido, y una de estas categorías se refiere a la mirada sobre el embarazo y el cuidado de las adolescentes embarazadas. Teniendo en cuenta que la visión del cuidado es siempre subjetiva y única, estas jóvenes demostraron que la forma en que son acogidas en la unidad de salud donde realizan el prenatal es el diferencial para iniciar su prenatal y continuar su seguimiento. Insertadas en un contexto de vulnerabilidades, muchas de estas adolescentes esperan, a través del embarazo, una forma de reconocimiento e inserción en la sociedad, de valoración y pertenencia, y expresan, de las más variadas formas, la preocupación y celo por sí mismas y por los demás. (En este caso el niño), siendo promotores de su proceso de atención junto con los profesionales de la salud que lo asisten.

Palabras clave: Adolescentes embarazadas; Cuidado de la salud; Atención básica; Vulnerabilidades; Anfitrión.

\section{Introdução}

Os adolescentes fazem parte de um grupo especial de atendimento, portanto necessitam de cuidado diferenciado, considerando os determinantes e condicionantes de saúde de sua faixa etária (Eisenstein, 2005). Eles devem ser acolhidos, independentemente do conhecimento ou presença dos pais ou responsáveis, em qualquer Unidade de Saúde, tendo seus direitos garantidos pelo Estatuto da Criança e Adolescente (ECA) (Brasil, 1990).

Uma questão sempre presente quando se trata de adolescentes, e de difícil manejo por muitos profissionais, é a gravidez na adolescência. Apesar da queda de $17 \%$ na taxa de natalidade em adolescentes nesta faixa etária, entre os anos de 2004 e 2015, a taxa de fecundidade no Brasil, ainda nesta mesma faixa etária, era considerada alta e refletia às características do contexto de desenvolvimento brasileiro, visto que as condições sociais que ainda prevalecem, são: renda, raça ou cor e escolaridade. Esses dados são relevantes "uma vez que adolescentes pobres, negras ou indígenas e com menor escolaridade tendem a engravidar mais que outras adolescentes" (Bruno, Carvalho \& Herlano, 2009). Portanto, trata-se de relevante pauta para o planejamento de ações em saúde a este grupo prioritário.

A Rocinha, local onde foi realizada a pesquisa, é um bairro localizado na zona sul da cidade do Rio de Janeiro, inserida em contextos sociais complexos, dentre eles: violência, pobreza e baixa escolaridade, os quais agravam a situação de vulnerabilidade e iniquidade dessas adolescentes. Durante as consultas de pré-natal realizadas nessa Unidade, localizada neste referido bairro, observou-se a singularidade e a dificuldade de comunicação e socialização da adolescente gestante, na visão da autora. Além disso, o absenteísmo às consultas de pré-natal e puerpério e/ou o início do pré-natal após a $12^{\mathrm{a}}$ semana de gestação é algo comum, e está inserido na prática diária dos serviços de saúde na APS. Os questionamentos surgiram à medida que foram evidenciadas as faltas destas adolescentes às consultas e a inquietude da pesquisadora de saber o que acontece no período em que essas meninas não comparecem à Unidade de Saúde para iniciar seu pré-natal.

O tema "gravidez na adolescência" frequentemente surge analisado sob a perspectiva do profissional de saúde, mas muito raramente na visão das adolescentes, enquanto detentoras de cuidado e saber. Enquanto que para os profissionais o saber que a adolescente traz, muitas vezes, passa despercebido, em detrimento ao próprio saber técnico. É consenso que o ato de "acolher" uma adolescente gestante e escutar suas percepções, em um primeiro contato, pode ter grande influência no processo de produção de cuidado em saúde. Segundo Omole-Ohonsi e Attah (2010, p. 318), "adolescentes que recebem boa assistência pré-natal, apoio financeiro, emocional e psicológico da família e da comunidade, tendem a apresentar resultados perinatais favoráveis".

Para que o processo de cuidado em saúde tenha impacto positivo neste grupo, estratégias que permitam minimizar desigualdades e/ou iniquidades e estigmas e/ou preconceitos, devem ser implementadas pelos profissionais de saúde que atuam com essa população específica, principalmente na Atenção Básica, que se caracteriza como porta de entrada e coordenadora do cuidado, ordenando as ações e serviços disponibilizados na rede de saúde. Formas inovadoras de atenção à saúde e caminhos para o cuidado integral, são retratados quando assumem, não somente o tratamento de doenças, mas a inclusão de pessoas em 
sistemas de produção de cuidados e de participação na afirmação da vida, centrada nas necessidades dos usuários (Brasil, 2005).

Desta forma, como ferramentas do cuidado, o acolhimento e o vínculo destacam-se favoravelmente. Vínculo e acolhimento refletem-se no cuidado em saúde. Para Cecílio e Merhy (2002), o somatório de um grande número de pequenos cuidados parciais forma o cuidado idealizado e, assim, vão se complementando, sendo negociado ou não entre o ser cuidador e o ser que é cuidado, mesclando-se até produzirem vida. Portanto, cuidado é vida. O cuidado é traduzido pela preocupação com o sujeito e também com alguns princípios, como saber ouvir, respeitar e acolher. Este tipo de cuidado deve ser ofertado pelos profissionais de saúde da atenção básica, a fim de criar um elo positivo entre o profissional e a gestante adolescente. O somatório de um grande número de pequenos cuidados parciais forma o cuidado idealizado e, assim, vão se complementando, sendo negociado ou não entre o ser cuidador e o ser que é cuidado, mesclando-se até produzirem vida. Portanto, cuidado é vida (Cecílio \& Merhy, 2003).

O ser humano é dotado de inúmeras atitudes de cuidado, enquanto ser relacional e de múltiplas interações, em seus aspectos físicos, psíquicos, social ou espiritual. O cuidado faz parte da existência humana como manifestação de compartilhamento, de troca e de reciprocidade e suas diferentes compreensões, demonstram a riqueza do cuidado no processo de viver do ser humano, isento de hierarquia (Backes et al., 2006).

O objetivo deste estudo, foi identificar o significado da gestação e do cuidado em saúde sob a ótica de adolescentes gestantes, durante o pré-natal, no contexto geográfico, cultural e social do bairro da Rocinha/RJ.

\section{Metodologia}

Trata-se de um estudo moldado em pesquisa qualitativa, exploratória, fundamentada nos delineamentos de pesquisa social. A pesquisa qualitativa pretende compreender o universo de significados, motivos, crenças, valores e atitudes dos sujeitos diante de uma determinada experiência vivida, bem como contribuir para a melhor compreensão da distância entre o conhecimento e a prática, na medida em que auxilia perceber os sentimentos das pessoas, explicando suas ações diante de uma dada situação (Minayo, 2010).

O cenário deste estudo foi a Unidade de Saúde da Família CMS Dr. Albert Sabin, localizada no bairro da Rocinha, no município do Rio de Janeiro, composta por seis equipes de ESF. Apesar de pertencerem ao mesmo território de abrangência, as equipes possuem características bem diferentes umas das outras. Existem áreas em que os moradores possuem água encanada, energia elétrica, casas em alvenaria, outras áreas possuem casas construídas com pedaços de latas, sem nenhuma infraestrutura e até pessoas que moram no interior de grutas ou em imóveis invadidos, por estarem abandonados. A Rocinha, antes denominada a maior favela do país, passou a ser considerada um bairro da Zona Sul do município do Rio de Janeiro, a partir do ano de 1993. A população da Rocinha, pelo Censo do Instituto Brasileiro de Geografia e Estatística (IBGE) de 2010, foi de 69.356 habitantes, com densidade domiciliar de 2,9 hab/dom (Ibge, 2010).

As participantes deste estudo foram 20 adolescentes gestantes, cadastradas na referida Unidade de Saúde, na faixa etária de 10 a 19 anos. Os critérios de inclusão: estar gestante na faixa etária entre 10 a 19 anos de idade, com pré-natal iniciado no CMS Dr. Albert Sabin, independente da idade gestacional, pertencentes a qualquer das seis equipes da ESF atuantes nessa Unidade. Os critérios de exclusão foram: adolescentes gestantes que residiam fora da área de abrangência do CMS Dr. Albert Sabin e que não realizavam o Pré-Natal na referida Unidade. Todas as participantes que atenderam aos critérios de inclusão foram convidadas a responder um roteiro de entrevista semiestruturado.

A pesquisa foi aprovada pelos Comitês de Ética e Pesquisa com Seres Humanos da Prefeitura do Rio de Janeiro/RJ e da Universidade Federal do Estado do Rio de Janeiro (UNIRIO), conforme Pareceres $\mathrm{n}^{\circ} 2.237 .447$ e $\mathrm{n}^{\circ}$ 2.267.506, 
respectivamente. As normas éticas para pesquisas que envolvem seres humanos foram rigorosamente seguidas, de acordo com o estabelecido na Resolução no 466/12 do Conselho Nacional de Saúde (Brasil, 2012).

A produção de dados ocorreu no período de março a maio de 2018, quando a unidade de saúde possuía um quantitativo de 161 gestantes, das quais 22 eram adolescentes na faixa etária de dez a 19 anos; ou seja, 13,5\% do total de gestantes atendidas na Unidade eram adolescentes e atendiam aos critérios de inclusão estabelecidos.

A participação na pesquisa foi de forma voluntária e as adolescentes e seus respectivos responsáveis foram esclarecidos quanto aos seus objetivos e aos procedimentos implicados na mesma. Foram disponibilizados o Termo de Consentimento Livre e Esclarecido (TCLE) para cada adolescente e para seus responsáveis, o Termo de Assentimento (TA), manifestando a sua concordância. As entrevistas foram realizadas na Unidade de Saúde, coincidindo com os dias agendados para a consulta pré-natal e, também, na residência da gestante. O quantitativo de adolescentes gestantes entrevistadas foi de 20 dessas, 16 tiveram seus dados coletados na Unidade de Saúde, em sala reservada para este fim e quatro, em suas respectivas residências. O tempo médio de duração das entrevistas foi de vinte minutos, sendo as entrevistadas identificadas de forma a garantir seu sigilo e anonimato, utilizando-se como codificação a letra G (Gestante) seguida de numeração sequencial arábica de 1 a 20 (Ex: G1, G2), conforme a realização das entrevistas.

Para análise dos dados, utilizou-se o método de análise de conteúdo, cumprindo as etapas da organização na modalidade temática (pré-análise, exploração do material e tratamento dos resultados; codificação; categorização, inferência) e onde foram identificadas 203 Unidades de Registro (UR), sendo relacionadas as palavras ou expressões que mais apareceram nas falas das adolescentes, separadas e agrupadas de acordo com os temas de Bardin e Oliveira (Bardin, 2004; Oliveira, 2008). Surgiram, então, 18 Unidades de Significação (US), que foram associadas e agrupadas, dando origem às pré-categorias e categorias. Como uma das categorias finais deste estudo, surgiu a visão sobre gravidez e cuidado das adolescentes gestantes.

\section{Resultados}

Os dados apresentados no quadro 1 abaixo foram extraídos das entrevistas realizadas com as adolescentes gestantes, correspondendo ao item I - Dados Pessoais do instrumento de coleta de dados.

Quadro 1 - Item I (dados pessoais).

\begin{tabular}{|c|c|c|c|c|c|}
\hline \multicolumn{6}{|c|}{ ITEM I: DADOS PESSOAIS } \\
\hline Idade ( $\mathrm{n}$ - gestantes) & Idade Gestacional & № Gravidezes & Frequentou escola & Escolaridade & Trabalham \\
\hline $\begin{array}{l}13(02) \\
15(03) \\
16(03) \\
17(02) \\
18(05) \\
19(05)\end{array}$ & $\begin{array}{l}10 \text { trimestre }(01) \\
20 \text { trimestre }(08) \\
30 \text { trimestre }(11)\end{array}$ & $\begin{array}{c}\text { 1ㅇ gestação (16) } \\
\text { 20 gestação ou mais } \\
(04)\end{array}$ & $\begin{array}{l}\text { SIM - } 12 \\
\text { NÃO - } 8\end{array}$ & $\begin{array}{l}\text { Ensino Fundamental I Completo - } 03 \\
\text { Ensino Fundamental I Incompleto - } 02 \\
\text { Ensino Fundamental II Completo - } 03 \\
\text { Ensino Fundamental II Incompleto - } 05 \\
\text { Ensino Médio Incompleto - } 07\end{array}$ & $\begin{array}{c}\text { SIM - } 3 \\
\text { NÃO - } 17\end{array}$ \\
\hline
\end{tabular}

Fonte: Elaborado pelos autores (2018).

A média de idade das adolescentes gestantes entrevistada foi de 16,3 anos. Existe uma forte relação entre gravidez em adolescentes e a iniciação precoce da sexualidade, famílias desestruturadas sem presença de diálogo com os pais (Melhado, Sant'anna, Passarelli \& Coates, 2008).

Dados da Organização das Nações Unidas para a Educação, Ciência e Cultura (UNESCO) mostram que as jovens vêm iniciando a vida sexual cada vez mais cedo, entre 15,2 e 16 anos, o que aponta para a relação entre iniciação sexual precoce e gravidez (United Nations, 2002).

Questões familiares, trabalho e gravidez são os três principais elementos que afastam as adolescentes brasileiras dos estudos, segundo uma pesquisa sobre evasão escolar feita em parceria com o Ministério da Educação, a Organização dos 
Estados Ibero-Americanos (OEI) e a Faculdade Latino-Americana de Ciências (FLACSO). Nesse estudo, 18,1\% das entrevistadas indicaram a gravidez como o principal motivo do abandono escolar (Abramovay, Castro, Silva \& Cerqueira, 2016). Desse modo, as adolescentes entrevistadas estão compatíveis com os dados desta pesquisa, pois, das oito (100\%) que estão fora da escola, duas (20\%) o fizeram por vergonha de continuar frequentando as aulas estando grávida. As demais $(80 \%)$ já não frequentavam a escola por outras causas (falta de apoio familiar e questões financeiras).

O abandono dos estudos não acontece pela rejeição das escolas à situação da gravidez, mesmo a grande parte delas não estão preparadas para receber e acompanhar estas adolescentes de maneira adequada. Acontece principalmente por sentimentos ambivalentes dessas meninas: vergonha sobre a exposição da sua sexualidade ou aceitação e satisfação pela gravidez. Os fatores emocionais, diversas vezes estão correlacionados à falta de estímulo dos próprios pais ou cuidadores, que valorizam mais o trabalho porque a jovem poderá ajudar na renda familiar, do que os estudos. Todavia, o fato de não concluírem a escolarização, traz dificuldades para alcançarem a independência financeira e profissional (Dadoorian, 2003).

A análise das entrevistas apontou para a visão subjetiva dessas adolescentes acerca do cuidado em saúde no pré-natal, sendo a subjetividade caracterizada como algo que varia de acordo com o julgamento de cada pessoa, consistindo em um tema que cada indivíduo pode interpretar à sua maneira. É aquilo que diz respeito ao sentimento pessoal, como a opinião sobre determinado assunto (Molon, 2003). É algo que muda de acordo com cada pessoa, como o gosto, sendo formada através das crenças e valores individuais, com suas experiências e histórias de vida.

\section{Discussão}

Foram destacados das falas das adolescentes, os sentimentos que surgem a partir da gestação. Sentimentos considerados positivos: aceitação como algo bom; felicidade; maturidade; valorização; pertencimento; normalidade. Sentimentos considerados negativos: indecisão; medo/desespero; culpa; tristeza; decepção; perda; desvalorização.

Exprimiram também sentimentos ambíguos, como a felicidade e a tristeza, a valorização e a desvalorização. De acordo com as entrevistadas, $75 \%$ considera a gravidez como algo bom, que acontece em um momento oportuno, apesar das incertezas. Uma forma de emergir de sua insegurança. As falas a seguir retratam esta questão:

Estar grávida é bom, para mim é bom, é uma coisa boa [...] eu sinto felicidade por ter uma criança mexendo dentro de mim. Eu falo com ele, ele mexe, então é uma felicidade (G2).

No começo achei bom, porque eu queria isso e não queria, e agora seja o que Deus quiser. Que seja tudo bem tanto para mim quanto para o meu bebê (G3).

Os outros $25 \%$ considera a gestação como algo ruim, que interfere negativamente em seu contexto de vida. A tristeza e a culpa por terem ficado grávidas na adolescência, também apareceram nas falas, com angústia e sofrimento interior aflorando nessas adolescentes. As mudanças no corpo são consideradas desagradáveis e a sensação de desespero no momento segue durante toda gestação.

Para mim estar grávida é horrível. Meu corpo tá horroroso, já engordei quase 15 quilos. Estou me sentindo péssima G13).

Para mim, estar grávida é um pesadelo. Eu só deixei uma vez e pronto, engravidei (G15).

Apesar das circunstâncias sociais desfavoráveis, o desejo de ter o filho foi predominante entre as adolescentes entrevistadas. A ocorrência de gravidez na adolescência é um fato rotineiro e comum entre o meio social em que convivem e 
algumas amigas, irmãs, primas ou a própria mãe são ou foram mães adolescentes. Também a personalidade e o comportamento dos adolescentes podem torná-los mais vulneráveis aos riscos do envolvimento com drogas, gravidez precoce e práticas ilícitas (Sierra \& Mesquita, 2006). Uma cultura de violência e medo, antes contida nas comunidades e agora disseminada em toda a sociedade, é o reflexo dos efeitos da exclusão de jovens adolescentes na contemporaneidade, que ora aparecem como vítimas, ora como autores de crimes e atos de vandalismos.

Ser adolescente nas favelas, significa lidar com a violência diariamente, com o preconceito em relação à pobreza e ao local de moradia, com a marginalização e o desamparo governamental (Castro \& Correa, 2005). O que se observa na atualidade, são adolescentes e jovens desamparados, vivendo um cotidiano repleto de dificuldades e com carências econômicas, sociais e de todo tipo de cuidado.

Existe uma valorização da maternidade neste caso, onde a menina assume um novo papel social: o de adolescentemãe-mulher, sendo a gravidez a via de acesso à feminilidade. A maternidade é, por excelência, uma experiência privada e que sempre foi atravessada pelo discurso sociopolítico em todas as épocas (Rena, Romagnol \& Lima, 2014). É, portanto, uma fase da vida da mulher que existe desde sempre, porém, por inúmeras vezes com características sociais importantes para compensação na sociedade na qual encontra-se inserida.

Minha prima que vem aqui, também de 14 anos, tá grávida, então na família tem nós duas grávidas. A gente vai poder cuidar das crianças juntas (G18).

A afirmação social nesse meio expressa-se na maternidade, o que possibilita dizer que se trata, nesse caso, de uma gravidez social, isto é, maternidade social. Através do filho, essas jovens sentem-se mães e mulheres (Dadoorian, 2003).

Porque quando eu estiver sozinha, não vou estar sozinha, vou ter uma companhia para conversar comigo, vai ser uma ótima companhia $(\mathrm{G} 2)$.

Estar grávida é a melhor coisa do mundo, uma coisa boa (G10).

Para mim a gravidez, veio na hora certa (G16).

Nos dias atuais, a gravidez na adolescência constitui-se uma questão polêmica. Correlaciona-se à aspectos relacionados ao exercício da sexualidade e da vida reprodutiva, às condições materiais de vida e às múltiplas relações de desigualdades presentes na vida social do país. Segundo Renepontes (2005), nesse sentido, é mais apropriado que a gravidez na adolescência seja vista como um ponto de mudança, que resulta de uma pluralidade de experiências de vida com diferentes significados, abordado de várias maneiras e com vários desfechos.

Para a compreensão do fenômeno da gravidez na adolescência, é fundamental considerar um conjunto de fatores causais que apontam para a necessidade de atenção integral e cuidado à saúde desse segmento, com enfoque na integração das políticas de saúde e de outras políticas sociais.

Os sentimentos de decepção, desvalorização e até mesmo culpa, surgem como algo que desagrada não somente à adolescente, mas à sua família; uma forma de punição transitória através da retirada de bens materiais ou afastamento sentimental, além de uma autopunição por ter rompido um elo de confiança:

Estar grávida é uma experiência nova, difícil, já perdi muitas coisas em questão de bens materiais, porque minha mãe cortou meu telefone e moto, enfim, é complicado (G6).

Fiquei com vergonha porque só eu na minha família tive a oportunidade de estar estudando e indo melhor, e agora sou uma decepção para eles (G14). 
Para mim a gravidez foi um susto, porque eu tinha acabado de ter uma festa de 15 anos, meu pai ficou louco (G16).

No início, a família da adolescente não reage favoravelmente à gravidez da filha, entretanto, após esse primeiro momento, aceitam esse fato e se posicionam da forma que acham ser adequada. A gravidez da jovem é vivida por toda a família, seja positivamente ou não, tendo no filho um traço de união entre eles.

Fiquei desesperada quando descobri que estava grávida, chorando, pois já tenho 1 filho de 3 anos, eu ficava o seguinte: eu não acredito, agora que meu filho saiu das fraldas, falando tudo, comendo tudo...vou voltar tudo de novo [...] então, fiquei bastante desesperada. Conversei muito com minha mãe, chorei muito (G7).

A descoberta e vivência da gravidez da adolescente, no âmbito familiar, torna-se um período de grandes transformações, com várias possíveis consequências, levando a família e a adolescente a refazerem seus projetos de vida (Nascimento, Xavier \& Sá, 2011):

Nesta idade dá um certo medo por ser a primeira, meio complicado, mudança de vida, de hábitos, a gente vai indo, tentando se acostumar (G9).

Mas agora tá tudo bem. Ele [o pai dela] meio que aceitou (G18).

O planejamento da gravidez com o parceiro, ou o desejo desta, implica em repensar a gravidez na adolescência como algo indesejado. Deste modo, a gestação na adolescência também aparece como uma forma de valorização e reconhecimento desta adolescente como mulher, pela sociedade à qual ela pertence, de acordo com as falas a seguir:

Estar grávida para mim foi muito bom. Eu e meu namorado tínhamos planejado tudo. Ele tem 18 anos e sempre quis muito ter um filho. E eu pude dar para ele o filho homem que ele tanto queria (G12).

Eu tava querendo mesmo, eи e meu namorado estávamos planejando (G16).

Para essas adolescentes, muitas vezes por terem uma precária situação econômica que lhes dificulta o acesso a bens de serviço e a serviços essenciais, projetam no filho a representatividade de seu "tudo". É uma forma de pensar que exprime um resgate pessoal projetado no filho, um sentido para a vida. Definem para si mesmas o desejo de proporcionar o melhor para eles, que eles estudem, trabalhem e que não lhes falte nada.

Uma coisa boa que parece que foi uma salvação porque eu estava no fundo do poço, na merda, e fez que eu saísse e me mostrasse e tomasse vergonha na cara, criasse maturidade, mostrar as coisas do mundo (G10).

Minhas amigas amaram, me disseram que vão me ajudar a cuidar. Meu namorado também tem 18 anos e a mãe dele disse que ele vai ter que trabalhar para comprar as coisas para o bebê. Mas eu amo tanto ele, acho que vamos conseguir (G20).

O entendimento do significado da palavra cuidado, para as entrevistadas, é caracterizado como: algo bom; correlacionado às equipes de saúde/unidades de saúde; serviços prestados como vacinação, consultas e demais procedimentos técnicos; atendimento em consultas pelos profissionais de saúde; prevenção de doenças; dedicação; amor; preocupação com o outro; atenção; carinho; estranheza; desconhecimento. 
A ESF surge no Brasil como principal estratégia política para a reorientação do modelo de atenção à saúde, fundamentando-se em uma nova ética social e cultural com vistas à promoção da saúde e da qualidade de vida dos indivíduos, famílias e comunidades (Sousa, 2008). As adolescentes entrevistadas reconhecem a Unidade Básica de Saúde (UBS) neste contexto, nos moldes da ESF, como promotora do cuidado e compreendem que a dimensão deste cuidado vai além dos procedimentos técnicos exercidos nas Unidades de Saúde.

A caracterização de cuidado pelas adolescentes entrevistadas é feita pela ideia de solidariedade, receptividade e uma postura acolhedora pelos profissionais de saúde. Para tanto, demanda-se do profissional algum nível de sensibilidade e de identificação com os problemas das pessoas, o que permite a expressão de sua subjetividade (Arce \& Sousa, 2013). A seguir, falas que exemplificam essa questão:

Cuidado é bom. Entendo que cuidado é que qualquer dúvida eu venho ao posto para tirar as dúvidas com meus médicos e na consulta (G1).

Cuidado é se prevenir de qualquer tipo de doença, se cuidar, sempre estar no posto de saúde (G2).

As referências que elas apresentam sobre o significado da palavra cuidado são distintas, algumas vezes abstratas, permeando em suas falas a correlação de cuidado com algo que é feito para o outro e pelo outro, uma forma de zelo ou carinho, porém, não considerando sua participação no autocuidado. Seguem-se as falas a respeito:

Cuidado é estar sempre observando, perguntando para ver se está bem, mesmo que não esteja, é um tipo de cuidado com as outras pessoas (G7).

É cuidar da criança, porque ela vai vir no mundo mas não sabe de nada (G11).

Eu acho que cuidado é uma coisa estranha (G13).

Eu acho que o cuidado é ir cuidando um do outro, com amor e carinho. É se preocupar se o outro tá bem ou mal (G20).

Mesmo sem perceber, a adolescente gestante é protagonista do cuidado em todas as formas: quando refere o cuidar de si mesma e do outro, quando indica, procura e reconhece a Unidade de Saúde em seu processo de cuidado. E neste caso, cuidar de si mesma e do bebê compreende ir às consultas de pré-natal, estar sempre na UBS, fazer os exames, estar sempre observando as mudanças que ocorrem em seu corpo e seus sentimentos confusos nesta fase.

Cuidado para mim é sempre estar no posto, me cuidando, fazendo os exames, e depois do meu bebê as vacinas e as consultas (G12).

Cuidado para mim é se cuidar, vir nas consultas (G15).

\section{Conclusão}

Através deste estudo foi possível captar, de forma singular, os sentimentos e razões pelos quais fizeram surgir os questionamentos sobre seus acompanhamentos (ou não) às consultas de pré-natal na UBS e também seus conhecimentos acerca da gravidez. Em cada entrevista, além dos dados palpáveis que serviram para a análise, o sentimento experimentado de ouvir uma história de vida, mesmo que em um contexto inóspito ao qual estas adolescentes estão inseridas, cercado de todos os tipos de violências, ao que elas mesmo referiram, foi excepcional e esclarecedor, permitindo também fazer repensar a prática dos serviços de saúde. 
Políticas públicas de saúde integradas para adolescentes, repercutem de forma positiva no crescimento e no desenvolvimento saudáveis desse grupo populacional, garantindo seus direitos e proteção. De acordo com as Diretrizes Nacionais para a Atenção Integral à Saúde de Adolescentes e Jovens na Promoção, Proteção e Recuperação da Saúde (Brasil, 2010), a contribuição para o pleno desenvolvimento de suas potencialidades, enquanto pessoas e cidadãs, ampliam a realização de suas escolhas de forma responsável e de acordo com seus projetos de vida.

Observa-se com este estudo que a gravidez na adolescência muitas vezes está associada a fatores psicossociais relacionados ao ciclo de pobreza e educação a que essas meninas estão inseridas. O desconhecimento de perspectivas de vida diferente do seu cotidiano influencia nesta tomada de decisão também quando decidem ser mãe, fazendo com que a gravidez, embora precoce, seja desejada e também sua única possibilidade de mudança de status de vida. Esta visão de mudança se deve ao fato de, na favela, a ascenção de menina para mulher ser pontuada pela gestação.

Contribuir para melhorar as formas de acolhimento e acompanhamento das adolescentes gestantes na Atenção Básica é uma das perspectivas deste estudo, através de capacitação e sensibilização dos profissionais de saúde, reorganizando os serviços e aprimorando-os, dentro das diretrizes de organização do Sistema Único de Saúde (SUS). Também fomentar novas produções científicas sobre cuidado no pré-natal de adolescentes, valorizando a visão das mesmas, inerentes em seus contextos sociais, especialmente aquelas em condições de maiores vulnerabilidades.

O estudo alcançou o objetivo proposto, que foi identificar o significado da gestação e do cuidado nestas adolescentes gestantes no contexto em que vivem, tendo como desdobramento viável a necessidade de capacitação, caso necessário, e sensibilização do profissional da APS que adentra a vida e questionamentos destas adolescentes. $\mathrm{O}$ atendimento proporcionado a essas adolescentes deve ser prestado por profissionais de saúde capacitados, uma vez que demanda cuidados específicos nesta faixa etária. Em se tratando de adolescentes gestantes em situação de vulnerabilidade, os profissionais devem, ainda, buscar estabelecer vínculos também com a família, com a finalidade de encontrar estratégias que possibilitem resolver suas demandas prioritárias de saúde (Brasil, 2007).

Também é necessário destacar a importância deste estudo para os profissionais da APS, que atuam diariamente com adolescentes, podendo avaliar a singularidade dos mesmos, sendo ponto de partida para novos estudos sobre cuidado em saúde e, neste grupo etário no qual existem tantas barreiras de acolhimento e atendimento a serem vencidas, visando a qualidade do acompanhamento e valorização do ser adolescente.

\section{Referências}

Abramovay, M., Castro, M. C., Silva, A. P. \& Cerqueira, L. (2016). Diagnóstico participativo das violências nas escolas: falam os jovens. Rio de Janeiro: FLACSO.

Arce, V. A. R. \& Sousa, M. F. (2013). Comprehensive care: social representations of family health teams in Distrito Federal, Brazil. Saúde Soc., 22(1), 10923. doi: https://doi.org/10.1590/S0104-12902013000100011.

Backes, D. S., Sousa, F. G. M., Mello, A. L. S. F., Erdmann, A. L., Nascimento, K. C. \& Lessmann, J. C. (2006). Care conceptions: an analysis of Ph.D. dissertations presented in a nursing graduate program. Texto Contexto Enferm, 15, 71-8.

Bardin, L. (2004). Análise de conteúdo. Lisboa: Edições 70.

Brasil. (1990). Lei $n^{\circ}$ 8.069, de 13 de julho de 1990. Dispõe sobre o Estatuto da criança e do adolescente, e dá outras providências. Brasília: Presidência da República. http://www.planalto.gov.br/ccivil_03/leis/18069.htm.

Brasil. (2005). Ministério da Saúde. Secretaria de Gestão do Trabalho e da Educação na Saúde, Departamento de Gestão da Educação na Saúde. Curso de formação de facilitadores de educação permanente em saúde: análise do contexto da gestão e das práticas em saúde. Brasília: Ministério da Saúde. https://bvsms.saude.gov.br/bvs/publicacoes/curso_facilitadores_unidade_pratica.pdf.

Brasil. (2007). Ministério da Saúde. Secretaria de Atenção à Saúde. Área de Saúde do Adolescente e do Jovem. Marco legal: saúde, um direito de adolescentes. Brasília: Ministério da Saúde. https://bvsms.saude.gov.br/bvs/publicacoes/07_0400_M.pdf.

Brasil. (2010). Ministério da Saúde. Secretaria de Atenção à Saúde. Núcleo Técnico da Política Nacional de Humanização. Acolhimento nas práticas de produção de saúde. Brasília: Ministério da Saúde. https://bvsms.saude.gov.br/bvs/publicacoes/acolhimento_praticas_producao_saude.pdf. 
Research, Society and Development, v. 10, n. 9, e9010917885, 2021

(CC BY 4.0) | ISSN 2525-3409 | DOI: http://dx.doi.org/10.33448/rsd-v10i9.17885

Brasil. (2012). Ministério da Saúde. Conselho Nacional de Saúde. Resolução no 466, de 12 de dezembro de 2012. Dispõe sobre pesquisa envolvendo humanos. Brasília: Ministério da Saúde. https://bvsms.saude.gov.br/bvs/saudelegis/cns/2013/res0466_12_12_2012.html.

Bruno, Z. V., Carvalho, A. A. A. \& Herlano, F. H. C. C. (2009). Mortalidade materna na adolescência. Rio de Janeiro: Revinter.

Castro, L. R \& Correa, J. (2005). Juventude contemporânea: perspectivas nacionais e internacionais. Rio de Janeiro: FAPERJ.

Cecílio, L. C. O. \& Merhy, E. E. (2003). A integralidade do cuidado como eixo da gestão hospitalar. Campinas: UNICAMP.

Dadoorian, D. (2003). Gravidez na adolescência: um novo olhar. Psicol ciênc prof., 23(1), 84-91. doi: https://doi.org/10.1590/S1414-98932003000100012.

Eisenstein E. (2005). Adolescência: definições, conceitos e critérios. Adolesc Saúde, 2(2), 1-2.

Instituto Brasileiro de Geografia e Estatística [IBGE]. (2010). População residente em domicílios particulares ocupados em aglomerados subnormais. Rio de Janeiro: IBGE.

Melhado, A., Sant'anna, M. J. C., Passarelli M. L. B. \& Coates, V. (2008). Teenage pregnancy: impact of the integral attention given to the pregnant teenager and adolescent mother as a protective factor for repeat pregnancy. Adolesc Saúde, 5(2), 45-51.

Minayo, M. C. S. (2010). Pesquisa social: teoria, método e criatividade. (29th ed). Petrópolis: Vozes.

Molon, S. I. (2003). Subjetividade e constituição do sujeito em Vygotski. Petrópolis: Vozes.

Nascimento, M. G., Xavier, P. F. \& Sá, R. D. P. (2011). Adolescentes grávidas: a vivência no âmbito familiar e social. Adolesc Saúde, 8(4), $41-7$.

Oliveira, D. C. (2008). Análise de conteúdo temático-categorial: uma proposta de sistematização. Rev enferm, Rio de Janeiro, 16(4), 569-76.

Omole-Ohonsi, A. \& Attah, R. A. (2010). Obstetric outcome of teenager pregnancy in Kano, North-western Nigéria. West African Journal of Medicine, 29(5), 318-22. doi: 10.4314/wajm.v29i5.68252.

Rena, A. C. C. B., Romagnol, R. C. \& Lima, N. L. (2014). Motherhood and femininity: a study regarding the subjectivity of neglectful mothers on social welfare. Rev Latino-Am Psicopatol Fundam., 7(4), 845-57.

Sierra, V. M. \& Mesquita, W. A. (2006). Vulnerabilidades e fatores de risco na vida de crianças e adolescentes. São Paulo em Perspec, $20,148-55$.

Sousa, M. F. (2008). The family health program in Brazil: analysis of access to basic care. Rev Bras Enferm, 61(2), 153-8. doi: https://doi.org/10.1590/S003471672008000200002

United Nations. (2002). AIDS: o que pensam os jovens? Políticas e práticas educativas. Brasília: UNESCO. 